\title{
A Comparative Study of the Diagnostic and Prognostic Value of Squamous Cell Carcinoma Antigen and Alfa-foeto Protien in Hepatocellular Carcinoma Before and After Therapeutic Intervention
}

Tarek Maged Al Sakty ${ }^{1}$, Hesham Hamdy Radwan ${ }^{1}$, Mohamed El Gharib Abou El Maaty ${ }^{2}$, Ahmed Samir Allam ${ }^{1}$, Ramy Samir Abd El Hamid, Mohamed Gamal Yousef ${ }^{1}$ *

1 Internal Medicine Department, Faculty of Medicine, Ain Shams University, 2 Radiology Department, Faculty of Medicine, Ain Shams University

* Corresponding Author: Mohamed Gamal Yousef, E-mail: m.gamal.yousef@gmail.com

\begin{abstract}
Background: hepatocellular carcinoma (HCC) is one of the most common malignancies worldwide and it is one of the major causes of death, because of its high frequency and poor prognosis. Hepatocellular carcinoma is now a common malignancy in Egypt which usually develops on top of liver cirrhosis secondary to viral infection, as hepatitis $\mathrm{C}$ viruses increased the risk of $\mathrm{HCC}$ in the Egyptian patients. Aim of the Work: was to verify the possibility of using the plasma squamous cell carcinoma antigen level as a tumor marker for hepatocellular carcinoma and to evaluate its prognostic value in management of HCC. Patients and Methods: the study included 60 subjects divided into three groups: group I was 30 patients with hepatocellular carcinomas, group II was 15 patients with liver cirrhosis and group III was 15 normal subjects serving as a control group. Results: the plasma SCCA level was significantly higher in group I patients (with HCC), than in the group II patients (cirrhosis) and control group. SCCA showed direct significant correlation with the most of laboratory data specially AST, INR, number and size of lesion. Conclusion: plasma SCCA is a sensitive and specific serum marker for the diagnosis of HCC and combination of AFP and SCCA in screening and diagnosis of HCC yielded a better sensitivity in diagnosis of HCC.
\end{abstract}

Keywords: Squamous Cell Antigen, Alfa-foeto Protien, Hepatocellular Carcinoma, Therapeutic Intervention

\section{INTRODUCTION}

Hepatocellular carcinoma (HCC) is the seventh most common malignancy and the second leading cause of cancer-related deaths worldwide. Globally, there are approximately 750000 new cases and 700000 deaths of HCC reported per annum ${ }^{(\mathbf{1})}$. Two well-known risk factors for HCC are chronic viral hepatitis $\mathrm{B}(\mathrm{HBV})$ and $\mathrm{C}(\mathrm{HCV})$, which account for $80 \%-90 \%$ of all HCC cases worldwide. Other risks for HCC include obesity, diabetes, vitamin D deficiency, aflatoxin B1 exposure, alcoholic and non-alcoholic liver cirrhosis. However, the underlying mechanism of HCC has not been entirely elucidated. Surgical resection and orthotopic liver transplantation are the best curative tools for the long-term survival of HCC patients. However, surgical resection is not feasible in more than $80 \%$ of HCC patients because of tumor location, tumor size or severity of the underlying liver disease. Only 5\%-15\% of HCC patients are potentially resectable ${ }^{(2)}$. The overall five-year survival rate in patients with HCC is very low, ranging from $5 \%$ to $9 \%$. The cumulative five-year recurrence rate is approximately $70 \%$ to $80 \%$ even after curative surgical resection. Recurrence after resection generally results in a high rate of mortality. Current methods for HCC diagnosis are classified into the following main categories: imaging [abdominal ultrasonography, contrast-enhanced computed tomography (CT) and magnetic resonance imaging (MRI)] and laboratory biomarker analysis [serum alpha-fetoprotein (AFP) levels, However, the diagnostic performance of imaging technologies is unsatisfactory, particularly for the diagnosis of small lesions and early-stage HCC ${ }^{(3)}$. AFP is the most commonly used tumor marker for HCC diagnosis and prognosis prediction, but the false negative rate using AFP level alone is as high as $40 \%$ for patients with early-stage HCC. AFP levels remain normal in $15 \%-30 \%$ of all the patients, even patients with

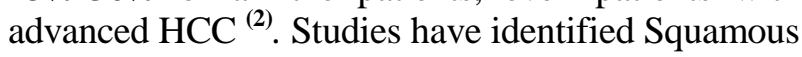
Cell Carcinoma Antigen (SCCA), as a potential novel HCC serum marker. Two genes, which share a high degree of homology (up to 98\%), encode for two $45 \mathrm{kDa}, 390$ amino acid-long proteins of the same name, which are $92 \%$ identical at the amino acid level. The first isoform, SCCA1, is neutral while the second isoform, SCCA2, is acidic. Importantly, a Third genetic variant of SCCA has also been identified in a minority of HCC cases, which is characterized by a G351A polymorphism in the Reactive core of the protein (4). Of significance is that serum (SCCA) is dramatically elevated in patients with $\mathrm{HCC}$, and the sensitivity and specificity of GP73 for HCC might be superior to those of AFP ${ }^{(5)}$. Trans arterial chemo 
embolization (TACE) and radio frequency ablation (RFA) are widely employed in patients with HCC, and has been recommended as the first choice for the middle to advanced stage HCC patients who cannot accept surgery. However, there is still lack of an effective way to evaluate the efficacy of TACE and RFA, whereas the changes of tumor size may not be reliable enough, and AFP is not sensitive ${ }^{(6)}$.

\section{AIM OF THE WORK}

The aim of this work is to evaluate the diagnostic value of serum level of Squamous Cell Carcinoma Antigen (SCCA) as a tumor marker for $\mathrm{HCC}$ and its prognostic value after trans arterial chemo embolization (TACE) or radiofrequency ablation (RFA), in comparison to alpha-feto protein (AFP).

\section{PATIENTS AND METHODS}

A- Patients: This study was performed on 60 subjects from the outpatient Hepatology clinic and inpatient Gastroenterology and Hepatology Department at Ain Shams University Hospital. They were classified into three groups: Group I: Thirty (30) patients with hepatocellular carcinoma who underwent either RFA or TACE. Group II: Fifteen (15) patients with liver cirrhosis without hepatocellular carcinoma. Group III: The control group composed of fifteen (15) age and sex matched healthy subjects. An informed consent was obtained from each patient before involvement in the study. The inclusion criteria for RFA: Age of 18-70 years, patients diagnosed with HCC based on the American Association for the Study of Liver Diseases (AASLD) guidelines in 2011 with an acceptable safe path between the tumor and the skin as shown on US ${ }^{(7)}$, patients with Child-Pugh A-B, a solitary HCC tumor $\leq 5 \mathrm{~cm}$ in diameter, or multiple HCC lesions $(\leq 3)$, each $\leq 3 \mathrm{~cm}$ in diameter, HCC that was visible on US, with an acceptable safe path between the tumor and the skin as shown on US. The Inclusion criteria for TACE: Age of 18-70, patients diagnosed with HCC based on the American Association for the Study of Liver Diseases (AASLD) guidelines in $2011^{(7)}$, patients with Child-Pugh A-B, large or multifocal HCC which were not suitable for surgical resection or radiofrequency ablation. Exclusion criteria for $\boldsymbol{R F}$ : Radiological evidence of invasion into the major portal or hepatic vein branches, patients with extrahepatic metastases, severe liver dysfunction Child-Pugh class $\mathrm{C}$, active gastrointestinal bleeding, pregnant ladies.
Exclusion criteria for TACE: Radiological evidence of invasion into the major portal or hepatic vein branches, patients with any other malignancies, severe liver dysfunction Child-Pugh class $\mathrm{C}$, active gastrointestinal bleeding, pregnant ladies, impaired coagulation functions (platelet count below $50 \times 109 / \mathrm{L}$ or prothrombin activity below 50\%). B-Methods: All subjects were subjected to the following: 1. Full history taking, 2. Full clinical examination, 3. Routine laboratory investigations, 4. Viral markers, 5. Serum Alpha fetoprotein, 6. Radiological study: Abdominal ultrasonography to assess the presence of liver cirrhosis, ascites and hepatic focal lesions, Tri phasic spiral CT abdomen: $\mathrm{CT}$ is done in different phases of contrast enhancement (early and late arterial and portal venous phases) it will be done for any patient showing a suspected focal lesion in the abdominal ultrasound, Dynamic MRI; if spiral $\mathrm{CT}$ is non conclusive. 7. Measurement of Serum Squamous Cell Carcinoma Antigen (SCCA) for all patients, 8. Follow up of the patients who had HCC and undergone either RFA or TACE will be done after 1 month by measuring serum level of alfa feto protein.

Statistical analysis: Data was analyzed using SPSS 16. Mean + standard deviation was computed for age, weight and height. Chi square test was applied to compare cardiovascular side effects. Repeated measures analysis of variance (ANOVA) was used to compare effects like heart rate, systolic and diastolic blood pressures of the two groups. P-value of 0.05 or less was considered statistically significant.

\section{RESULTS}

Table (1): Comparison between the three groups regarding age

\begin{tabular}{|c|c|c|c|c|c|c|}
\hline \multirow{2}{*}{ Groups } & \multicolumn{4}{|c|}{ Age } & \multicolumn{2}{|c|}{ ANOVA } \\
\hline & \multicolumn{2}{|c|}{ Range } & \multicolumn{2}{|c|}{\begin{tabular}{|l|l|} 
Mean & SD \\
\end{tabular}} & \multirow[t]{2}{*}{$\mathbf{F}$} & \multirow[t]{2}{*}{ P-value } \\
\hline Group I & 39 & -67 & \begin{tabular}{|l|l}
$54.733 \pm$ \\
\end{tabular} & 7.634 & & \\
\hline Group II & 49 & -68 & $59.867 \pm$ & 6.232 & \multirow[t]{2}{*}{11.372} & \multirow[t]{2}{*}{$<0.001 *$} \\
\hline Group III & 34 & -58 & $47.333 \pm$ & 7.403 & & \\
\hline \multicolumn{7}{|c|}{ TUKEY'S Test } \\
\hline \multicolumn{3}{|c|}{ I\&II } & I\&III & & \multicolumn{2}{|c|}{ II\&III } \\
\hline \multicolumn{3}{|c|}{0.074} & $0.006^{*}$ & & \multicolumn{2}{|c|}{$<0.001 *$} \\
\hline
\end{tabular}




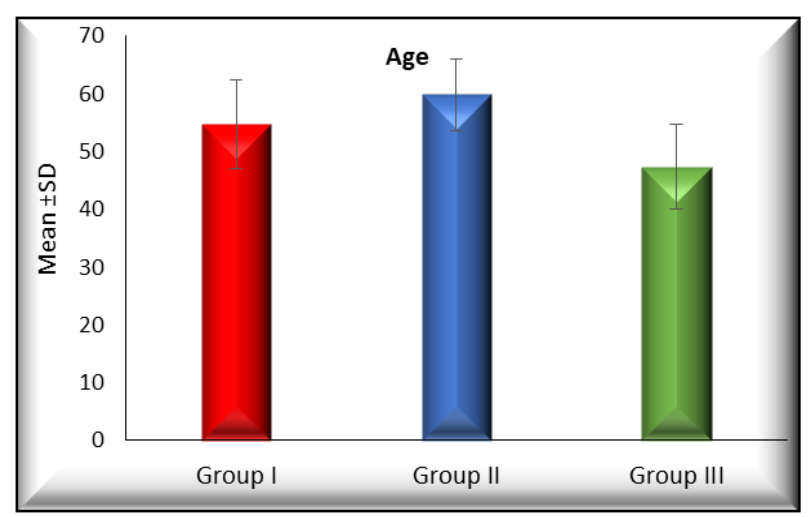

The table and graph show statistically significant difference between the groups I\&III and groups II\&III regarding age, but the difference was insignificant between groups I\&II.

Table (2): Comparison between the three groups regarding total bilirubin

\begin{tabular}{|c|c|c|c|c|c|}
\hline \multirow{2}{*}{ Groups } & \multicolumn{3}{|c|}{ T.Bil. } & \multicolumn{2}{|c|}{ ANOVA } \\
\hline & \multicolumn{2}{|c|}{ Range } & \begin{tabular}{|l|l} 
Mean & SD \\
\end{tabular} & \multirow[t]{2}{*}{$\mathbf{F}$} & \multirow[t]{2}{*}{ P-value } \\
\hline Group I & 1.5 & 3 & $2.053 \pm 0.393$ & & \\
\hline Group II & 0.7 & 2.3 & \begin{tabular}{|l|l|l|l}
1.447 & \pm 0.49 \\
\end{tabular} & \multirow[t]{2}{*}{47.355} & \multirow[t]{2}{*}{$<0.001 *$} \\
\hline Group III & 0.7 & 1.2 & $0.913 \pm 0.14 \mathrm{C}$ & & \\
\hline \multicolumn{6}{|c|}{ TUKEY'S Test } \\
\hline \multicolumn{2}{|c|}{ I\&II } & & I\&III & \multicolumn{2}{|c|}{ II\&III } \\
\hline \multicolumn{3}{|c|}{$<0.001 *$} & $<0.001 *$ & \multicolumn{2}{|c|}{$0.001 *$} \\
\hline
\end{tabular}

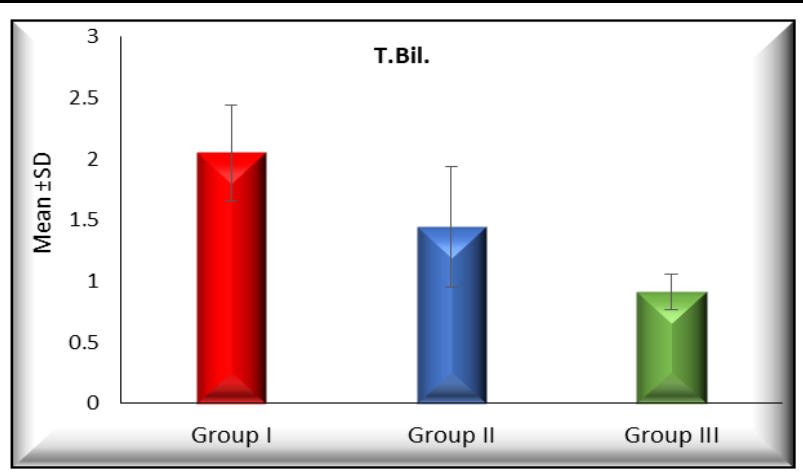

The table and graph show statistically significant difference between the three groups regarding the value of total bilirubin.

Table (3): Comparison between the three groups regarding direct bilirubin

\begin{tabular}{|c|c|c|c|c|c|}
\hline \multirow{2}{*}{ Groups } & \multicolumn{3}{|c|}{ D.Bil. } & \multicolumn{2}{|c|}{ ANOVA } \\
\hline & \multicolumn{2}{|c|}{ Range } & Mean \pm SD & \multirow[t]{2}{*}{$\mathbf{F}$} & \multirow[t]{2}{*}{ P-value } \\
\hline Group I & 0.8 & 1.8 & $1.100 \pm 0.268$ & & \\
\hline Group II & 0.3 & 1.2 & \begin{tabular}{|l|l|}
0.713 & \pm 0.267 \\
\end{tabular} & \multirow{2}{*}{33.992} & \multirow[t]{2}{*}{$<0.001 *$} \\
\hline Group III & 0.3 & -0.7 & \begin{tabular}{|c|c|}
$0.493 \pm 0.153$ \\
\end{tabular} & & \\
\hline \multicolumn{6}{|c|}{ TUKEY'S Test } \\
\hline \multicolumn{3}{|c|}{ I\&II } & I\&III & \multicolumn{2}{|c|}{ II\&III } \\
\hline \multicolumn{3}{|c|}{$<0.001 *$} & $<0.001 *$ & \multicolumn{2}{|c|}{$0.044 *$} \\
\hline
\end{tabular}

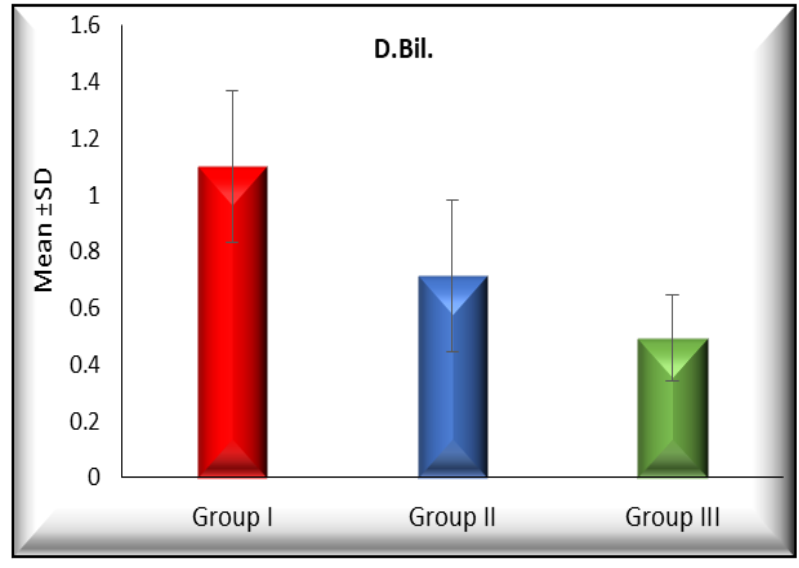

The table and graph show statistically significant difference between the three groups regarding the value of direct bilirubin.

Table (4): Comparison between group I and group II regarding Child Score

\begin{tabular}{|c|c|c|c|c|c|c|c|c|}
\hline \multirow{3}{*}{ Child } & \multicolumn{6}{|c|}{ Groups } & \multirow{2}{*}{\multicolumn{2}{|c|}{ Chi-Square }} \\
\hline & \multicolumn{2}{|c|}{ Group I } & \multicolumn{2}{|c|}{ Group II } & \multicolumn{2}{|c|}{ Total } & & \\
\hline & $\mathbf{N}$ & $\%$ & $\mathbf{N}$ & $\%$ & $\mathbf{N}$ & $\%$ & $\mathbf{X}^{2}$ & P-valu \\
\hline hild A & 0 & 0.00 & 6 & 40.00 & 6 & 13.33 & \multirow{4}{*}{15.416} & \multirow{4}{*}{$<0.001 *$} \\
\hline Child B & 9 & 30.00 & 5 & 33.33 & 14 & 31.11 & & \\
\hline Child C & 21 & 70.00 & 4 & 26.67 & 25 & 55.56 & & \\
\hline otal & 30 & 100.00 & 15 & 100.00 & 60 & 100.00 & & \\
\hline
\end{tabular}

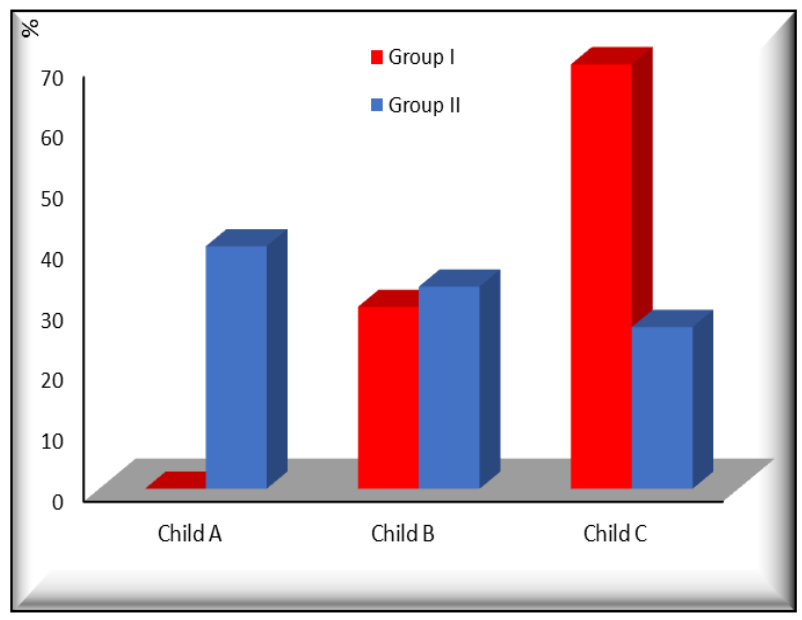

The table and graph demonstrate statistically significant difference between groups (I\&II) regarding child score.

Table (5): Comparison between the three groups regarding presence of $\mathrm{HCV} \mathrm{Ab}$

\begin{tabular}{|c|c|c|c|c|c|c|c|c|}
\hline \multirow{3}{*}{$\mathrm{HCV}$ Ab } & \multicolumn{6}{|c|}{ Groups } & \multirow{2}{*}{\multicolumn{2}{|c|}{ Chi-Square }} \\
\hline & \multicolumn{6}{|c|}{\begin{tabular}{|l|l|l|} 
Group I & Group II Group III & Total \\
\end{tabular}} & & \\
\hline & \begin{tabular}{|l|l|}
$\mathbf{N}$ & $\%$ \\
\end{tabular} & \begin{tabular}{l|l}
$\mathbf{N}$ & $\%$ \\
\end{tabular} & $\mathbf{N}$ & $\%$ & 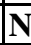 & $\%$ & $\mathbf{X}^{2}$ & P-value \\
\hline Negative & \begin{tabular}{l|l|l}
11 & 36.67 \\
\end{tabular} & \begin{tabular}{|l|l|}
5 & 33.33 \\
\end{tabular} & 15 & 100.00 & & 51.67 & & \\
\hline sitive & 963.33 & \begin{tabular}{l|l|}
1066.67 \\
\end{tabular} & 0 & 0.00 & & 48.33 & 8. & $<0.001 *$ \\
\hline Total & 30100.00 & $15 \mid 100.00$ & & 100.00 & & 00.00 & & \\
\hline
\end{tabular}




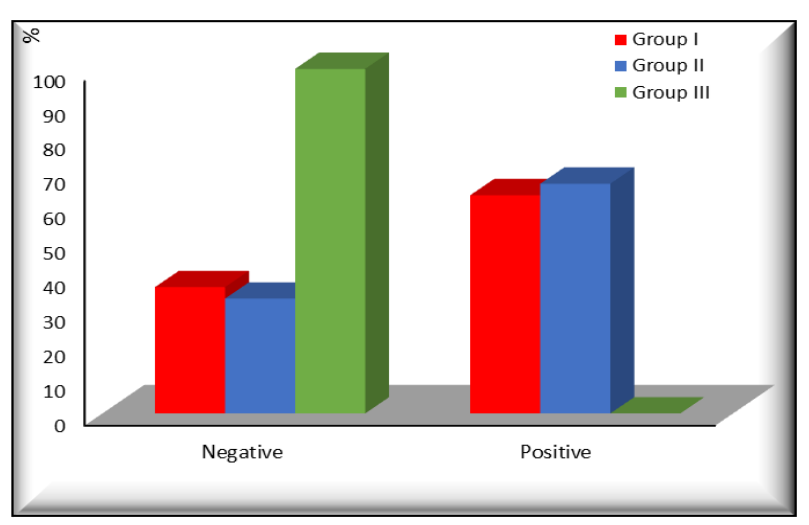

The table and graph indicate statistically higher incidence of $\mathrm{HCV} \mathrm{Ab}$ in group one and group two than in the controls.

Table (6): Comparison between the three groups regarding pre intervention values for SCCA

\begin{tabular}{|c|c|c|c|c|c|}
\hline \multirow{2}{*}{ Groups } & \multicolumn{3}{|c|}{ SCCA Pre } & \multicolumn{2}{|c|}{ ANOVA } \\
\hline & \multicolumn{2}{|c|}{ Range } & \begin{tabular}{|l|l|} 
Mean \pm SD \\
\end{tabular} & \multirow[t]{2}{*}{$\mathbf{F}$} & \multirow{2}{*}{ P-value } \\
\hline Group I & 3.1 & 12.1 & $8.213 \pm 2.486$ & & \\
\hline Group II & 1.2 & 6 & \begin{tabular}{|l|l|l}
2.807 & 1.447 \\
\end{tabular} & \multirow[t]{2}{*}{72.607} & \multirow[t]{2}{*}{$<0.001 *$} \\
\hline Group III & 0.4 & 3 & $\mid 1.607 \pm 0.761$ & & \\
\hline \multicolumn{6}{|c|}{ TUKEY'S Test } \\
\hline \multicolumn{3}{|c|}{ I\&II } & I\&III & \multicolumn{2}{|c|}{ II\&III } \\
\hline \multicolumn{3}{|c|}{$<0.001 *$} & $<0.001 *$ & \multicolumn{2}{|c|}{0.219} \\
\hline
\end{tabular}

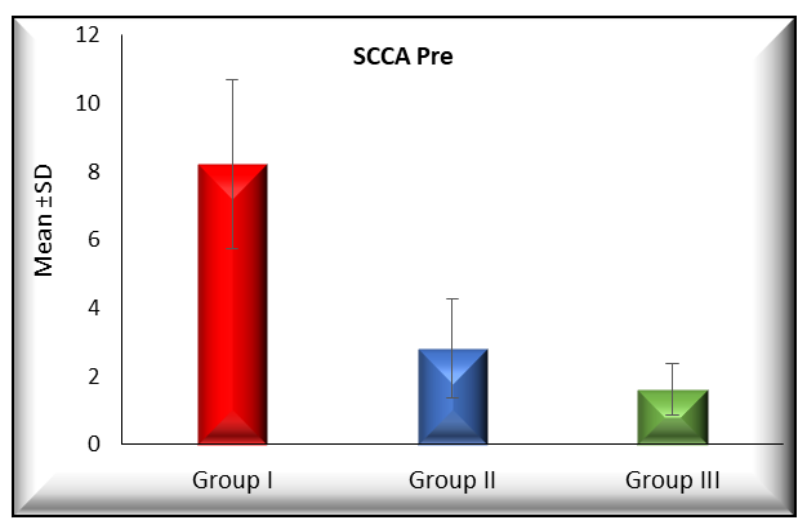

The table and graph reveal statistically significant difference between groups (I\&II) and groups (I\&III) regarding the pre intervention values of SCCA, but the difference between groups (II\&III) was insignificant.

\section{DISCUSSION}

Hepatocellular carcinoma (HCC) is one of the commonest cancers worldwide. It is a major health problem and its incidence is increasing. The presence of cirrhosis of the liver is the major risk factor and worldwide this is largely due to chronic hepatitis $\mathrm{C}$ virus (HCV) and hepatitis B virus
(HBV) infection ${ }^{(\boldsymbol{8})}$. The development of $\mathrm{HCC}$ is closely associated with cirrhosis. HCC is accompanied by liver cirrhosis in $72 \%$ of patients (9). Currently, serum a-fetoprotein (AFP), a fetalspecific glycoprotein, has undoubtedly been the most widely used tumor marker for the detection and monitoring of HCC. However, serum AFP is not always elevated to a diagnostic level in all patients, particularly in small HCC, and considerable numbers of patients with more advanced stages would be missed unless another diagnostic tool is used ${ }^{(\mathbf{1 0})}$. Moreover, its level may be elevated in non-malignant chronic liver diseases, including chronic hepatitis and cirrhosis, as well as in other primary and secondary liver cancers. Therefore, the identification of alternative serum markers of HCC is needed ${ }^{(\mathbf{1 1})}$. The AASLD recommends surveillance of adults with cirrhosis because it improves overall survival, and it suggests surveillance using ultrasound (US), with or without alpha-fetoprotein (AFP), every 6 months (12). SCC-Ag is a subfraction of the tumorassociated antigen TA-4, and it is a $45-\mathrm{kDa}$ glycoprotein. Increased serum SCC-Ag levels are observed in squamous cell carcinoma of the esophagus, lung, head and neck, and cervix ${ }^{(\mathbf{1 3})}$. The aim of this study is to assess the serum level of Squamous cell carcinoma antigen and alpha fetoprotien in patients with liver cirrhosis and those with hepatocellular carcinoma and to compare them with normal subjects to detect their sensitivity and specificity as a diagnostic marker. Also to study the clinical significance of serum SCCA in hepatitis $\mathrm{C}$ cirrhotic patients with $\mathrm{HCC}$ as a prognostic marker before and after intervention to improve the outcome of HCC diagnosis and treatment. This study was carried on 60 subjects classified into 3 groups: Group I was 30 patients with hepatocellular carcinoma and liver cirrhosis who were subjected to intervention wether TACE or RFA, Group II was 15 patients with liver cirrhosis only without HCC, Group III was 15 healthy subjects served as control group. In the present study the age of HCC patients ranged from 39 to 67 years with a mean \pm SD of $54.7 \pm 7.6$ years and this is probably attributed to the duration of the underlying liver disease, HCC is reported to develop in the fifth decade. The same results were reported by Johnson (14), who found that the average age of patients ranged from fifth to sixth decades of life, and it was also showed a significant 
familial correlation of onset age of hepatocellular carcinoma between probands and their relatives. Familial HCC cases have earlier age of onset and their relatives have a higher $\mathrm{HCC}$ risk in an early age. Although AFP -the only serological marker currently available in clinical practice- is not a sufficiently reliable marker to identify HCC patients, mainly because of its poor sensitivity and elevation in non-malignant liver disease, but its slope increasing greater than $7.5 \mathrm{ng} / \mathrm{mL}$ per month despite locoregional therapy is associated with post-LT HCC recurrence and may serve as a surrogate for microvascular invasion. These findings support incorporating changes in the AFP into candidate selection for LT ${ }^{(\mathbf{1 5})}$. In this study we found that combining AFP and SCCA yielded a better sensitivity in diagnosis of HCC than using each marker alone and this is in agreement with Zhang et al. ${ }^{(16)}$ who confirmed the efficiency of SCCA as a marker for HCC by studying the possibility of combining AFP and SCCA for better serological detection of HCC. Also these results were not comparable to those obtained by Wan et al. ${ }^{(17)}$ who found that the sensitivity of AFP was $66 \%$ and the specificity was $95 \%$. The sensitivity and specificity of SCCA has been shown to vary with the different cutoff values used. According to these results the sensitivity and specificity of SCCA for selective detection of the HCC group over the cirrhotic group were $93.33 \%$ and $96.67 \%$ respectively, at a cut-off value of $5.1 \mathrm{ng} / \mathrm{mL}$ these results were comparable to those of Pozzan et al. (18) who found that SCCA sensitivity was $89 \%$ and specificity was $50 \%$ at a cut off value of 130 $\mathrm{AU} / \mathrm{mL}$. The obvious difference in specificity value is probably attributed to difference in sample size and difference in ethnicity, but they weren't in accordance to the values mentioned by Bellissimo et al. ${ }^{(19)}$ who found SCCA had a sensitivity of $42 \%$ and specificity of $83 \%$ using a cut-off of 3.8 $\mathrm{ng} / \mathrm{mL}$. The SCCA1 was not elevated in patients with HCC arising on top of nonalcoholic and alcoholic fatty liver disease which suggests that this HCC marker may be etiology related and so results are different in Egyptian patients as most HCC here arise on top of HCV and/or HBV liver disease. Also, the choice of optimal biomarkers for HCC surveillance may be determined by the etiology of underlying liver disease. In conclusion SCCA expression was significantly higher in cirrhosis and HCC than in group II. This proves that SCCA may play role in the progression of liver disease and hepatocarcinogenesis. The results obtained in this study will be valuable for the future application of plasma SCCA as a routine biomarker for the detection of $\mathrm{HCC}$ in patients with hepatitis $\mathrm{C}$ virus related liver cirrhosis. Also the marked significant reduction of serum SCCA levels in HCC patients subjected to either RF ablation or TACE proved that SCCA may play a prognostic marker in HCC management especially that SCCAIgM levels $(\mathrm{P}=0.004)$ was an independent predictor of survival according to Zhang et al. ${ }^{(16)}$.

\section{CONCLUSION}

Plasma SCCA is a sensitive and specific serum marker for the diagnosis of $\mathrm{HCC}$ and combination of AFP and SCCA in screening and diagnosis of HCC yielded a better sensitivity in diagnosis of HCC.

\section{CONFLICTS OF INTEREST}

There are no conflicts of interest.

\section{REFERENCES}

1. Xu L, Xiaocong W, Pujun G (2017): Diabetes Mellitus and Risk of Hepatocellular Carcinoma Biomed Res Int., 5202684.

2. Zhang J, Shao C, Zhou Q, Zhu Y, Zhu J and Tu C (2015): Diagnostic accuracy of serum squamous cell carcinoma antigen and squamous cell carcinoma antigen-immunoglobulin $\mathrm{M}$ for hepatocellular carcinoma: A meta-analysis. Molecular and Clinical Oncology, 3(5): 1165-1171.

3. Ji J, Wang H, Li Y, Zheng L, Yin Y, Zou Z, Zhou F, Zhou W, Shen F and Gao C (2016): Diagnostic evaluation of desgamma-carboxy prothrombin versus $\alpha$ fetoprotein for hepatitis B virus-related hepatocellular carcinoma in China: a large-scale, multicentre study. PloS one, 11(4): 153-227.

4. Giannelli G, Marinosci F, Trerotoli P, Volpe A, Quaranta $M$ and Dentico $P$ (2005): SCCA antigen combined with alpha-fetoprotein as serologic markers of HCC. International Journal of Cancer, 117:506-509. 
5. Soyemi O, Otegbayo J, Ola S, Akere A, Soyemi $T$ (2012): Comparative diagnostic efficacy of serum squamous cell carcinoma antigen in hepatocellular carcinoma. BMC Res Notes, 5: 403.

6. Chok KS, Chan MM, Dai WC, Chan AC, Cheung TT, Wong TC, She WH and Lo CM (2017): Survival outcomes of hepatocellular carcinoma resection with postoperative complications-a propensity-score-matched analysis. Medicine, 96(12).

7. Bruix J and Sherman M (2011): American Association for the Study of Liver Diseases. Management of hepatocellular carcinoma: an update. Hepatology, 53: 1020.

8. Ryerson AB, Eheman CR, Altekruse SF, Ward JW, Jemal A, Sherman RL, Henley SJ, Holtzman D, Lake A, Noone AM and Anderson RN (2016): Annual Report to the Nation on the Status of Cancer, $1975-2012$, featuring the increasing incidence of liver cancer. Cancer, 122(9): 1312-1337.

9. Jemal A, Bray F, Center MM, Ferlay J, Ward E, Forman D (2011): Global cancer statistics. CA Cancer J Clin., 61: 69-90.

10. Stefaniuk P, Cianciara J and WiercinskaDrapalo A (2010): Present and future possibilities for early diagnosis of hepatocellular carcinoma. World journal of gastroenterology: WJG., 16(4): 418.

11. Nishida N, Arizumi T, Hayaishi S, Takita M, Kitai S, Yada N, Hagiwara S, Inoue T, Minami Y, Ueshima $K$ and Sakurai $\mathbf{T}$ (2012): Gender differences in the livers of patients with hepatocellular carcinoma and chronic hepatitis $\mathrm{C}$ infection. Digestive Diseases, 30(6): 547-553.

12. Singal AG, Pillai A, Tiro J (2014): Early detection, curative treatment, and survival rates for hepatocellular carcinoma surveillance in patients with cirrhosis: a meta-analysis. PLoS Med., 11:e1001624.
13. Oh J and Bae JY (2018): Optimal cutoff level of serum squamous cell carcinoma antigen to detect recurrent cervical squamous cell carcinoma during posttreatment surveillance. Obstetrics \& Gynecology Science, 61(3): 337-343.

14. Johnson PJ, Berhane S, Kagebayashi C, Satomura S, Teng M, Reeves HL, O'Beirne J, Fox R, Skowronska A, Palmer D and Yeo W (2015): Assessment of liver function in patients with hepatocellular carcinoma: a new evidencebased approach - the ALBI grade. Journal of Clinical Oncology, 33(6): 550.

15. Giannelli G, Fransvea E, Trerotoli P, Beaugrand M, Marinosci F, Lupo L, Nkontchou G, Dentico P and Antonaci $S$ (2007): Clinical validation of combined serological biomarkers for improved hepatocellular carcinoma diagnosis in 961 patients. Clinica Chimica Acta., 383(1-2): 147-152.

16. Zhang TT, Zhao XQ, Liu Z, Mao ZY and Bai L (2016): Factors affecting the recurrence and survival of hepatocellular carcinoma after hepatectomy: a retrospective study of 601 Chinese patients. Clin Transl Oncol., 18: 831-840.

17. Wan HG, Xu H, Gu YM, Wang H, Xu W and Zu MH (2014): Comparison osteopontin vs AFP for the diagnosis of HCC: a meta-analysis. Clinics and Research in Hepatology and Gastroenterology, 38(6): 706-714.

18. Pozzan C, Cardin R, Piciocchi M, Cazzagon N, Maddalo G, Vanin V, Giacomin A, Pontisso P, Cillo $U$ and Farinati F (2014): Diagnostic and prognostic role of SCCA - IgM serum levels in hepatocellular carcinoma (HCC). Journal of Gastroenterology and Hepatology, 29(8): 1637-1644.

19. Bellissimo F, Pinzone MR, Cacopardo B and Nunnari G (2015): Diagnostic and therapeutic management of hepatocellular carcinoma. World Journal of Gastroenterology, 21(42): 12003. 